\title{
Involving service users in trials: developing a standard operating procedure
}

\author{
Bridie Angela Evans ${ }^{1 *}$, Emma Bedson², Philip Bell ${ }^{3}$, Hayley Hutchings ${ }^{1}$, Lesley Lowes ${ }^{4}$, David Rea ${ }^{5}$, Anne Seagrove ${ }^{1}$, \\ Stefan Siebert ${ }^{6}$, Graham Smith ${ }^{3}$, Helen Snooks ${ }^{1}$, Marie Thomas ${ }^{7}$, Kym Thorne ${ }^{1}$, lan Russell ${ }^{1}$ and on behalf of the \\ West Wales Organisation for Rigorous Trials in Health (WWORTH)
}

\begin{abstract}
Background: Many funding bodies require researchers to actively involve service users in research to improve relevance, accountability and quality. Current guidance to researchers mainly discusses general principles. Formal guidance about how to involve service users operationally in the conduct of trials is lacking. We aimed to develop a standard operating procedure (SOP) to support researchers to involve service users in trials and rigorous studies.

Methods: Researchers with experience of involving service users and service users who were contributing to trials collaborated with the West Wales Organisation for Rigorous Trials in Health, a registered clinical trials unit, to develop the SOP. Drafts were prepared in a Task and Finish Group, reviewed by all co-authors and amendments made.

Results: We articulated core principles, which defined equality of service users with all other research team members and collaborative processes underpinning the SOP, plus guidance on how to achieve these. We developed a framework for involving service users in research that defined minimum levels of collaboration plus additional consultation and decision-making opportunities. We recommended service users be involved throughout the life of a trial, including planning and development, data collection, analysis and dissemination, and listed tasks for collaboration. We listed people responsible for involving service users in studies and promoting an inclusive culture. We advocate actively involving service users as early as possible in the research process, with a minimum of two on all formal trial groups and committees. We propose that researchers protect at least $1 \%$ of their total research budget as a minimum resource to involve service users and allow enough time to facilitate active involvement.
\end{abstract}

Conclusions: This SOP provides guidance to researchers to involve service users successfully in developing and conducting clinical trials and creating a culture of actively involving service users in research at all stages. The UK Clinical Research Collaboration should encourage clinical trials units actively to involve service users and research funders should provide sufficient funds and time for this in research grants.

Keywords: Consumer participation, Consumer involvement, Clinical trials, Service user involvement

\section{Background}

Actively involving service users in health and social care research is encouraged as a way to improve research quality, relevance and accountability [1-3]. The Department of Health and devolved administrations, notably the Welsh Government, recommend it as good practice. Many health research funding bodies now require information on their application forms about the extent and methods of involving service users in designing and

\footnotetext{
* Correspondence: b.a.evans@swansea.ac.uk

${ }^{1}$ College of Medicine, Swansea University, Swansea, Wales, UK

Full list of author information is available at the end of the article
}

undertaking research [4-6] (http://www.hta.ac.uk/public/ getinvolved/conductingresearch.shtml or http://www.sdo. nihr.ac.uk/getinvolved.html). The Central Commissioning Facility of the National Institute for Health Research states on its website that "applications that are technically excellent but have little patient or public involvement may be asked to address this before an offer of funding is made" (http://www.nihr-ccf.org.uk/site/ consumerinvolvement/infoforresearchers/default.cfm).

Service users bring understanding and experience of conditions and interventions to the process of designing and conducting trials [5,7]. We use the term 'service user'

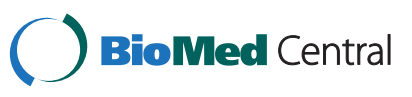


to refer to patients, carers and people eligible for a service, whether or not identified or diagnosed, and anyone else considered relevant to the study inclusion criteria [8].

Reports of impacts include influencing the development of research studies (e.g. shaping research questions); the choice of methods for trials (e.g. by helping to select the content of control arms); making the research more ethical and encouraging recruitment (e.g. by helping explain different concepts in patient information to enable informed consent); widening dissemination (e.g. by identifying nonacademic routes and the best format for communicating findings) [3,9-17]. Involving service users can increase public understanding of the purpose and process of science and can help make researchers more accountable to the people who may use services and contribute through taxes and donations [3].

Service users contribute to both the content and methods of research, including developing, reviewing and commissioning research proposals and undertaking research using a wide range of methods [13,18-26]. Health Technology Assessment International (HTAi) has a Citizen and Patient Involvement group that aims to embed public and patient involvement across its membership of 59 countries (see http://www.htai.org/index. php?id=545) and public involvement in international Health Technology Assessment activities is growing [27]. Work is underway to enhance the reporting of public and patient involvement in health research through GRIPP 2 with EQUATOR to strengthen the evidence base about service user involvement in research (see http://www.equator-network.org/resource-centre/ library-of-health-research-reporting/reporting-guidelines/ other-reporting-guidelines/). Although researchers are increasingly involving service users in planning and undertaking research, there is limited engagement with clinical trials $[3,28]$.

Guidance on how to involve service users in research mainly discusses general principles (e.g. [29]). Recent guidance by INVOLVE outlines benefits and challenges of engaging service users in trials [30]. However, formal guidance about how to involve service users operationally in the conduct of trials is lacking although guidance for service users has recently been prepared (see www. trialsjournal.com/imedia/1292821605618248/supp1.doc). Recent evidence reports the importance of context and process on involvement, highlighting the need for researchers to understand their role in facilitating service user involvement and follow best practice [13,31]. Researchers can be unsure how to involve people in research and need access to information and support in order to understand the potential opportunities and how to manage the involvement process with clarity and consensus [32]. There is also a need to tackle misunderstood anxieties around who to involve, to explain the contribution of service users' perspectives, so that researchers are not misled by anxieties about gaining representation, the so-called 'red herring' [33], in place of experience [32,34,35].

The West Wales Organisation for Rigorous Trials in Health (WWORTH) is a clinical trials unit based in Swansea University; it has recently received provisional UK Clinical Research Collaboration (UKCRC) registration following, among other things, the development of a suite of Standard Operating Procedures (SOPs). Although UKCRC did not require an SOP on involving service users before awarding registration, we judged that such an SOP would enhance researchers' ability to involve service users throughout development and implementation of trials and other rigorous studies. We believed it would provide legitimacy for involving service users in trials and provide a starting point on which researchers could build. We considered it would also benefit the conduct and general quality of such studies. Other WWORTH SOPs, now numbering more than 30 , cover project management, qualitative methods, the use of routine data and more familiar topics like randomisation, data management and statistical analysis.

Academics and service users together developed the SOP for involving service users to provide guidance to researchers. The authors have considered how to involve service users actively in trials and rigorous mixed-methods studies, and in developing research ideas through trial development groups. Using our experience, we aimed to ensure that the involvement of service users was in line with best practice and maximised opportunities to improve the design, conduct, analysis and dissemination of research $[36,37]$. We adhered to elements of the GRIPP checklist where relevant to reporting guidance [38].

\section{Methods}

Researchers at Swansea University with experience in actively involving service users joined one of three Task and Finish (TAF) groups responsible for developing WWORTH's initial portfolio of SOPs. Conscious that 'patient involvement' can be passive, even tokenistic, we chose to entitle our SOP 'Involving service users'. Thereafter the SOP followed a standard process: selected TAF members drafted the SOP in collaboration and other group members reviewed and amended successive drafts. Three service users with experience of contributing to trials and three academics with experience of actively involving service users in research, including trials, then reviewed the penultimate draft, leading to further amendments. Two members of the WWORTH Development Group, which coordinated the registration application to UKCRC, independently reviewed the final draft. The Development Group approved the SOP for use following discussion at a regular meeting. The SOP development process is shown in Table 1. 
Table 1 Process of developing the SOP

\begin{tabular}{ll}
\hline Key steps & Who was involved \\
\hline Step 1: drafting SOP & Researchers with experience of involving service users in research + Clinical Trial Unit staff \\
Step 2: reviewing draft SOP & Service users + external academics with experience of involving service users in research \\
Step 3: approving SOP & Service users + researchers with experience of involving service users in research + Clinical Trial Unit staff \\
\hline
\end{tabular}

We based this SOP on research evidence, good practice and experience of actively involving service users in research, including randomised trials [9,20,39-42]. We aimed to define a process that addressed service users' needs [43-45] and also ensured their partnership within the research process from the development of a proposal and involvement as co-applicants, through undertaking the study, to disseminating results in a range of formats.

For us, the term 'involvement' epitomises the process of actively involving service users within the research process and taking full consideration of their contributions at all stages of trial development and implementation, interpretation of results and dissemination. This is in line with INVOLVE's definition of public involvement in research as research being carried out 'with' or 'by' members of the public rather than 'to', 'about' or 'for' them [36]. Involvement is different from participation, where people take part in a research study, and engagement, where information and knowledge about research are provided and disseminated [36]. We expected that service users would collaborate and share decisions with other partners. Collaboration is defined as an approach characterised by partnership between researchers and members of the public and shared decision-making. It is the second level of INVOLVE's involvement continuum (consultation collaboration - user controlled). We also described opportunities for involvement by consultation to inform a process of collaborative or shared decision-making since involvement in a project can vary between approaches $[32,36,37]$. The SOP therefore assumes service users are involved in trials through collaboration and consultation.

\section{Results}

We articulated the key principles that underpinned the SOP:

- Service users' knowledge is a valuable contribution to the formulation of evidence-based medicine and should contribute to trials whenever possible

- Service users should contribute at all stages of a trial, from germination of an idea to dissemination of results

- Service users should have equal status with other research team members for the expertise they bring; all team members' views should receive equal consideration

- Like all team members, service users should receive the information and practical assistance necessary to undertake their role
We recommend that trial development groups formalise these principles in their Terms of Reference when starting to involve service users within a trial team to articulate shared and differing motivations and vision. The SOP, which can be viewed at www.trustresearch.org.uk/ en/publications.htm, also provides guidance on how to achieve these key principles, divided into three sections. Each element of the SOP links back to source evidence, allowing the reader to gauge provenance and seek further information.

\section{SOP guidance}

\section{Section 1: planning the process of involving service users}

We advise that researchers understand the benefits of involving service users in trials and how to do so. The reasons for involving service users and the research aims and objectives should inform decisions on who to involve and how to identify them through patient groups or charities, either focusing on the condition in question or generic Public and Patient Involvement groups and voluntary associations. We highlight practical ways of enabling involvement like: reimbursement of costs; payment of honoraria; selection of suitable venues; appropriate meeting arrangements and information formats; training and support for service users; and provision of sufficient time, expertise and financial and other resources to enable service users to contribute successfully to trials. We propose that researchers protect a minimum of $1 \%$ of their total research budget to support service users and build sufficient additional time into proposed timescales to allow for careful recruitment and active involvement of service users. This equates to $£ 10,000$ of a $£ 1$ million trial or $£ 20,000$ of a $£ 2$ million trialThis should be allocated for expenses to include honorariums to take part in meetings, out of pocket expenses, the provision of training, support and other costs to facilitate service users' full involvement over the life of a trial.

\section{Section 2: implications for trial management of involving service users}

We list the people responsible for involving service users within trials and promoting a collaborative culture based on mutual respect, courtesy and equal opportunity. We ask the Chief Investigator to take general responsibility for implementing the SOP across the trial, and the Trial Manager or Trial Coordinator to take specific responsibility for supporting and liaising with the recruited 
service users. The SOP recognises other models exist and could be considered to support service users in trials, such as costing (for example, 5\%WTE) and naming a 'service user involvement lead'. The SOP advocates that research staff should have an understanding of how to support service users and provide training in this topic if necessary. This training should complement training to enable service users to contribute to research. Training for all trial members should be itemised within the trial budget. Additionally, Clinical Trial Units (CTU) should ensure staff are trained in service user involvement to undertake their CTU roles. We also advocate engaging service users as early as possible in the research process and including a minimum of two people on all formal trial groups and committees, in line with recognised good practice $[2,6,29]$. We recommend recruiting a third service user who also receives training and regular briefings in order to remain engaged and well informed and can cover for inevitable absences. In this way, the research team will maximise the likelihood that two service users can be effectively involved throughout the trial. Table 2 lists the roles and status of service users. We require service users to contribute to decisions about all aspects of planning, designing, conducting and reporting a trial, such as selecting outcomes, designing data collection tools, reviewing ethical standards, undertaking analysis and disseminating beyond academic audiences, as detailed in Figure 1. We ask that trial meetings and documents avoid acronyms and clinical terms where possible to facilitate equal participation of all and that Service Users be a standing agenda item to ensure that research teams hear and address relevant issues.

\section{Section 3: processes for involving service users in trials}

Figure 1 displays our flowchart for involving service users in research, derived from the Medical Research Council's framework for developing and evaluating complex interventions in health [7]. At each stage, we define a minimum level of involvement and propose additional opportunities such as specific service user groups, collaborative workshops and open meetings to enable service users to contribute at a level of detail and engagement not always feasible in trial development and management groups. In doing so, all co-authors recognise that different service users can best participate in different ways in order to bring to the research a range of perspectives based on different sources of information and evidence. Hence we seek to provide adequate opportunities for experiences that are relevant to the individual trial to be shared within the research team.

\section{Discussion}

Effectively involving service users benefits research [3]. In practice, however, seeking to involve service users encounters practical issues and unreceptive attitudes $[22,32,46]$. While SOPs cannot themselves overcome these barriers, or prevent tokenistic 'involvement', they can provide an appropriate framework, guide researchers about the processes required for good practice, and help to create a culture that expects to involve service users at all stages of the research process and is conducted to ethical standards $[28,47]$. Despite the emerging evidence base and strong policy support [48], the legitimacy of involving service users in research is still questioned and it still happens in a minority of research studies $[49,50]$. Our SOP recognises the moral justification for, and benefits of, including service users. It describes an expected minimum standard and culture to guide researchers to involve service users in trials and encourage them to further involvement. It also makes specific recommendations for providing the financial and time resources necessary to achieve effective involvement and foster an environment to support researchers in understanding its potential and current limitations $[10,19,43-45,51]$. It delineates the role of service users across all the structures and functions of a trial and thus establishes them as equal and legitimate members of a research team. Implementation of our SOP will enable the guidance to be tested and reviewed.

This SOP proposes that research teams allocate at least $1 \%$ of proposed trial financial budgets to the training and

Table 2 Role and status of service users at each trial management level

\begin{tabular}{lll}
\hline Level of management & Service user role & Service user involvement \\
\hline Trial Steering Committee (TSC) & $\begin{array}{l}\text { Contribute to corporate decision-making about design, } \\
\text { conduct and reporting }\end{array}$ & WILL involve two service users \\
$\begin{array}{ll}\text { Data Monitoring and Ethics } \\
\text { Committee (DMEC) }\end{array}$ & $\begin{array}{l}\text { Contribute to corporate decision-making about safety, } \\
\text { benefit and recruitment }\end{array}$ & SHOULD involve two service users but not mandatory \\
Trial Development Group (TDG) & $\begin{array}{l}\text { Contribute to corporate decision-making about all } \\
\text { aspects of proposed trial }\end{array}$ & WILL involve two service users \\
Trial Management Group (TMG) & Contribute to corporate decision-making about all & WILL involve two service users \\
Trial Research Team (TRT) & Collaborate in generic teamwork across trial & SHOULD involve two service users \\
Ad hoc operational meetings & Collaborate in task-oriented team work across trial & SHOULD involve two service users when feasible \\
\hline
\end{tabular}


support of service users and ensure that they have enough time within their busy schedules to provide effective training and moral support to enable collaboration. Funding bodies should expect proposed budgets and timetables to cover these additional resources, respond sympathetically to research proposals that do so and ensure that research awards allocate sufficient funds and time to underpin the effective involvement of service users.

\section{Conclusion}

Intention to involve service users actively in trials and other rigorous studies in health and social care is an explicit requirement in all major research funding bids and has become an essential feature of good research practice. We offer this SOP to guide researchers in successfully involving service users in developing and conducting clinical trials. We propose that UKCRC should require all clinical 
trial units seeking registration or re-registration to show how they will involve service users in their work by preparing a specific SOP within their portfolio of SOPs. We also propose that funding bodies should encourage researchers to build sufficient funds and time into research proposals to involve service users effectively. These steps will encourage good practice in involving service users in research and help to improve relevance, accountability and quality of health and social care research.

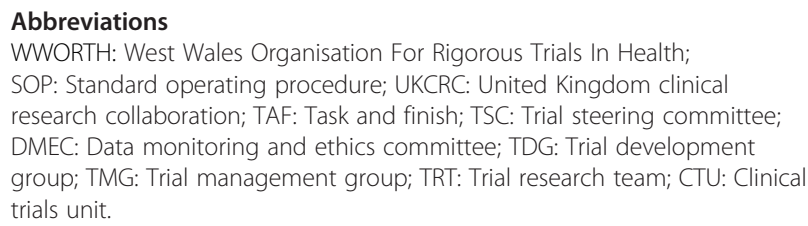

\section{Competing interests}

The authors declare that they have no competing interests.

\section{Authors' contributions}

BAE wrote the first draft of the Standard Operating Procedure and was responsible for revisions. $H H, I R$, AS, SS and MT were members of the authorship team. EB, PB, LL, DR, GS, HS and KT reviewed and commented on the SOP. BAE wrote the first draft of this manuscript and was responsible for the revisions. All authors provided input into drafting the manuscript and read and approved the final version.

\section{Acknowledgements}

We are grateful to Dr Daphne Russell for reviewing the SOP during its development and to Mel Storey, WWORTH Quality Assurance Officer, for managing the process of developing and approving the SOP.

\section{Author details}

${ }^{1}$ College of Medicine, Swansea University, Swansea, Wales, UK. ${ }^{2}$ Clinical Trials Research Centre, University of Liverpool, Liverpool, UK. ${ }^{3}$ Service user, Anglesey, Wales, UK. ${ }^{4}$ School of Nursing and Midwifery Studies, Cardiff University, Cardiff, Wales, UK. ${ }^{5}$ College of Human and Health Sciences, Swansea University, Swansea, Wales, UK. ${ }^{6}$ College of Medical, Veterinary and Life Sciences, University of Glasgow, Glasgow, UK. ${ }^{7}$ Faculty of Education and Social Services, University of Wales, Newport, Wales, UK.

Received: 12 December 2012 Accepted: 3 July 2013

Published: 17 July 2013

\section{References}

1. Beresford P: User involvement in research: exploring the challenges. Nursing Times Research 2003, 8:36-46.

2. Department of Health: Strengthening accountability: involving patients and the public: policy guidance, section 11 of the Health and Social Care Act. London: Department of Health; 2001.

3. Staley K: Exploring Impact: Public involvement in NHS, public health and social care research. Eastleigh: INVOLVE; 2009.

4. INVOLVE: Senior Investigators and Public Involvement. Eastleigh: INVOLVE; 2009.

5. O'Donnell M, Entwistle V: Consumer involvement in research projects: the activities of research funders. Health Policy 2004, 69:229-238.

6. Wales Office of Research and Development for Health and Social Care: Research Governance Framework for Health and Social Care in Wales: Second Edition. Cardiff: Welsh Assembly Government; 2005.

7. Craig N, Dieppe P, Macintyre S, Michie S, Nazareth I, Petticrew M: Developing and evaluating complex interventions: the new Medical Research Council guidance. Br Med J 2008, 337:979-983.

8. Buckland S, Hayes H, Ostrer C, Royle J, Steel R, Tarpey M, Walton J, Yeeles P: Public Information Pack: How to get actively involved in NHS, public health and social care research. Eastleigh: INVOLVE; 2007.

9. Simmons RK, Ogilvie D, Griffin SJ, Sargeant LA: Applied public health research - falling through the cracks? BMC Publ Health 2009, 9:362.
10. Trivedi $P$, Wykes $T$ : From passive subjects to equal partners: qualitative review of user involvement in research. Br J Psychiatry 2002, 181:468-472

11. Viswanathan M, Ammerman A, Eng E, Gartlehner G, Lohr KN, Griffith D, Rhodes S, Samuel-Hodge C, Maty S, Lux L, Webb L, Sutton SF, Swinson T, Jackman A, Whitener L: Community-Based Participatory Research: Assessing the Evidence. In Evidence Report/Technology Assessment No 99 AHRQ Publication 04-E022-2. Rockville, MD: Agency for Healthcare Research and Quality; 2004

12. Lowes L, Robling MR, Bennert K, Crawley C, Hambly H, Hawthorne K, Gregory JW, Team TDS: Involving lay and professional stakeholders in the development of a research intervention for the DEPICTED Study. Heal Expect 2010. doi:10.1111/j.1369-7625.2010.00625.x.

13. Brett J, Staniszewska S, Mockford C, Herron-Marx S, Hughes J, Tysall C, Suleman R: Mapping the impact of patient and public involvement on health and social care research: a systematic review. Heal Expect 2012. doi:10.1111/j.1369-7625.2012.00795.x.

14. Evans J, Rose D, Flach C, Csipke E, Glossop H, McCrone P, Craig T, Wilkes T: VOICE: Developing a new measure of service users' perceptions of inpatient care, using a participatory methodology. J Ment Heal 2012, 21(1):57-71.

15. Thompson J, Bissell P, Cooper C, Armitage C, Barber R: Credibility and the 'professionalized' lay expert: Reflections on the dilemmas and opportunities of public involvement in health research. Health 2012, 16:602-618

16. Barber R, Beresford P, Boote J, Cooper C, Faulkner A: Evaluating the impact of service user involvement on research: a prospective study. Int $J$ Consum Stud 2011, 35:609-615.

17. Callard F, Rose D, Wykes T: Close to the bench as well as at the bedside: involving service users in all phases of translational research. Heal Expect 2011. doi:10.1111/j.1369-7625.2011.00681.x.

18. Chalmers I: A guide to patient-led good controlled trials. Lancet 2000, 356:774

19. Clough R, Green B, Hawkes H, Raymond G, Bright L: Older People as Researchers: Evaluating a Participative Project. Joseph Rowntree Foundation; 2006. Available at: http://www.jrforg.uk/publications/older-peopleresearchers-evaluating-participative-project (Accessed 16 July 2013).

20. Hanley B, Truesdale A, King A, Elbourne D, Chalmers I: Involving consumers in designing, conducting and interpreting randomised controlled trials: questionnaire survey. Br Med J 2001, 322:519-523.

21. O'Donnell M, Entwistle V: Consumer involvement in decisions about what health-related research is funded. Health Policy 2004, 70:281-290.

22. Rhodes $P$, Nocon A, Wright J, Harrison S: Involving patients in research. Setting up a service users' advisory group. J Manag Med 2001, 15:167-171.

23. Smith E, Donovan S, Beresford P, Manthorpe J, Brearley S, Sitzia J, Ross F: Getting ready for user involvement in a systematic review. Heal Expect 2009, 12:197-208.

24. Boote J, Baird W, Sutton A: Involving the public in systematic reviews: a narrative review of organisational approaches and eight case examples. J Comp Effect Res 2012, 1(5):1-12.

25. Gillard S, Simons L, Turner K, Lucock M, Edwards C: Patient and public involvement in coproduction of knowledge: Reflection on the analysis of qualitative data in a mental health study. Qual Heal Res 2012, 22(8):1126037.

26. Katz M, Archer L, Peppercorn J, Kereakoglow S, Collyar D, Burstein H, Schilsky R, Partridge A: Patient advocates' role in clinical trials. Cancer 2012. doi:10.1002/cncr.27485.

27. Hailey D, Werko S, Bakri R, Cameron A, Gohlen B, Myles S, Pwu J, Yothasamut J: Involvement of consumers in the HTA activities of INAHTA members Report on a survey. Available at http://www.htai.org/index.php? id=745\#c2784 (downloaded 13 April 2013

28. Boote J, Baird W, Sutton A: Public involvement in the design and conduct of clinical trials: a review. Int J Interdiscip Soc Sci 2011, 5:91-112.

29. Steel R: Brief Summary and Checklist for Researchers, Research Commissioners and Research Groups for Involving Vulnerable and Marginalised People. Eastleigh: INVOLVE; 2003.

30. INVOLVE: Public involvement in clinical trials: Supplement to the briefing notes for researchers. Eastleigh: INVOLVE; 2012.

31. Staley K, Buckland SA, Hayles H, Tarpey M: 'The missing links': understanding how context and mechanism influence the impact of public involvement in research. Heal Expect 2012. doi:10.1111/hex.12017.

32. Boote J, Telford R, Cooper CL: Consumer involvement in health research: a review and research agenda. Health Policy 2002, 61:213-236. 
33. Beresford P: What stakeholders need to know about patients' views and experiences: no date. Available at http://webcache.googleusercontent.com/ search?q=cache:6JkdgX49AucJ:www.icms.qmul.ac.uk/chs/Docs/25214.pdf+\&cd= 1\&hl=en\&ct=clnk\&gl=uk (Accesssed: 13 April 2013).

34. Entwistle VA, Renfrew MJ, Yearley S, Forrester J, Lamont T: Lay perspectives: advantages for health research. BMJ 1998, 316:463-466.

35. Wright D, Corner J, Hopkinson J, Foster C: Listening to the views of people affected by cancer about cancer research: an example of participatory research in setting the cancer research agenda. Heal Expect 2006, 9:3-12.

36. INVOLVE: Briefing notes for researchers: public involvement in NHS, public health and social care research. Eastleigh: INVOLVE; 2012.

37. Boote J, Barber R, Cooper CL: Principles and indicators of successful consumer involvement in NHS research: Results of a Delphi study and subgroup analysis. Health Policy 2006, 75:280-297.

38. Staniszewska S, Brett J, Mockford C, Barber R: The GRIPP checklist: strengthening the quality of patient and public involvement reporting in research. Int J Technol Assess Health Care 2011, 27(4):391-399.

39. Snooks H, Cheung W-Y, Close J, Dale J, Gaze S, Humphreys I, Lyons R, Mason S, Merali Y, Peconi J, Phillips C, Phillips J, Roberts S, Russell I, Sanchez A, Wani M, Wells B, Whitfield R: Support and Assessment for Fall Emergency Referrals (SAFER 1) trial protocol. Computerised onscene decision support for emergency ambulance staff to assess and plan care for older people who have fallen: evaluation of costs and benefits using a pragmatic cluster randomised trial. BMC Emergency Medicine 2010, 10. http://www.biomedcentral.com/1471-227X/10/2.

40. Service Users Research Endeavour (SURE) Group: Patients' information sheets and multicentre studies. Lancet 2010, 376:514-515.

41. Edwards V, Wyatt K, Logan S, Britten N: Consulting parents about the design of a randomized controlled trial of osteopathy for children with cerebral palsy. Heal Expect 2011. doi:10.1111/j.1369-7625.2010.00652.x.

42. Snooks H, Anthony R, Chatters R, Cheung WY, Dale J, Donohoe R, Gaze S, Halter M, Koniotou M, Logan P, Lyons R, Mason S, Nicholl J, Phillips C, Phillips J, Russell I, Siriwardena AN, Wani M, Watkins A, Whitfield R, Wilson L: Support and assessment for fall emergency referrals (SAFER 2) research protocol: cluster randomised trial of the clinical and cost effectiveness of new protocols for emergency ambulance paramedics to assess and refer to appropriate community-based care. BMJ Open 2012, 2:e002169. doi:10.1136/bmjopen-2012-002169.

43. Nolan M, Hanson E, Grant G, Keady J, Magnusson L: Introduction: what counts as knowledge, whose knowledge counts? Towards authentic participatory enquiry. In User Participation in Health and Social Care Research. Edited by Nolan M, Hansen E, Grant G, Keady J. Maidenhead: OUP McGraw Hill; 2007:1-13.

44. Nolan M, Hanson E, Grant G, Keady J: Conclusions: realising authentic participatory enquiry. In User Participation in Health and Social Care Research. Edited by Nolan M, Hansen E, Grant G, Keady J. Maidenhead: OUP McGraw Hill; 2007:183-202.

45. Telford R, Boote JD, Cooper CL: What does it mean to involve consumers successfully in NHS research? A consensus study. Heal Expect 2004, 7:209-220.

46. Staniszewska S, Jones N, Newburn M, Marshall S: User involvement in the development of a research bid: barriers, enablers and impacts. Heal Expect 2007, 10:173-183.

47. INVOLVE: Patient and public involvement in research and research ethics committee review. Eastleigh: INVOLVE; 2009.

48. McLaughlin H: Service User Research in Health and Social Care. London: Sage; 2009

49. Kreis J, Puhan M, Schunemann H, Dickersin K: Consumer involvement in systematic reviews of comparative effectiveness research. Heal Expect 2012. 10.1111/j.1369-7625.2011.00722.x

50. Vale C, Thompson L, Murphy C, Forcat S, Hanley B: Involvement of consumers in studies run by the Medical Research Council (MRC) Clinical Trials Unit: Results of a survey. Trials 2012, 13:9.

51. Cooper D: Consumers in Canadian Arthritis Research. EULAR Health Professionals News 2008, 9(2):14-15.

doi:10.1186/1745-6215-14-219

Cite this article as: Evans et al:: Involving service users in trials: developing a standard operating procedure. Trials 2013 14:219.

\section{Submit your next manuscript to BioMed Central and take full advantage of:}

- Convenient online submission

- Thorough peer review

- No space constraints or color figure charges

- Immediate publication on acceptance

- Inclusion in PubMed, CAS, Scopus and Google Scholar

- Research which is freely available for redistribution

Submit your manuscript at www.biomedcentral.com/submit
C BioMed Central 